\title{
Tunneling conductance of a mesoscopic ring with spin-orbit coupling and Tomonaga-Luttinger interaction
}

\author{
M. Pletyukhov, ${ }^{1}$ V. Gritsev, ${ }^{2,3}$ and N. Pauget ${ }^{1}$ \\ ${ }^{1}$ Institut für Theoretische Festkörperphysik, Universität Karlsruhe, D-76128 Karlsruhe, Germany \\ ${ }^{2}$ Department of Physics, Harvard University, Cambridge, Massachusetts 02138, USA \\ ${ }^{3}$ Département de Physique, Université de Fribourg, CH-1700 Fribourg, Switzerland
}

\begin{abstract}
We study the tunneling current through a mesoscopic two-terminal ring with spin-orbit coupling, which is threaded by a magnetic flux. The electron-electron interaction in the ring is described in terms of a Tomonaga-Luttinger model which also allows us to account for a capacitive coupling between the ring and the gate electrode. In the regime of weak tunneling, we describe how, at temperatures lower than the mean level spacing, the peak positions of the conductance depend on magnetic flux, spin-orbit coupling strength, gate voltage, charging energy, and interaction parameters (charge and spin velocity and stiffness).
\end{abstract}

PACS numbers: 73.23.Ad, 71.70.Ej

\section{INTRODUCTION}

Mesoscopic rings represent an important tool for experimental and theoretical studies of various phenomena which take place on a submicrometer scale. The ring geometry allows one to probe many interesting theoretical predictions. One of the most exciting phenomena is the generation of geometric phases which are manifested in the interference patterns of wave packets propagating in the ring. Along with the well-known AharonovBohm (AB) effect $\frac{1}{\underline{1}}$ which takes place for both spinless and spinful particles, the generation of a spin-dependent phase is also possible. This effect, sometimes called the Aharonov-Casher (AC) effect ${ }^{2}$, may occur in the transport of electrons when they are subject to sufficiently strong spin-orbit (SO) coupling. The recent fabrication of $\mathrm{HgTe}$ rings ${ }^{3}$ made it possible to directly observe the AC phase. In earlier experiments with other compounds $\frac{4,5.6}{6}$ the signatures of this effect have been also detected.

In order to probe the $\mathrm{AC}$ phase it is necessary to have a tool for manipulating the strength of the spin-orbit coupling. This is provided by the gate-voltage dependence ${ }^{7}$ of the Rashba SO coupling ${ }^{8}$, which serves as a basis for a construction of a spin field-effect transistor ${ }^{9}$. Changing the magnetotransport properties of the ring in this way, the experimentalists are now able to study the $\mathrm{AC}$ effect ${ }^{3.4}$.

Usually the current through a mesoscopic noninteracting ballistic ring is described theoretically by means of the Landauer-Büttiker scattering matrix theory ${ }^{10}$. Geometric phases arising due to both magnetic flux and SO coupling can be naturally incorporated in this formalism 11.12.13.14.15. Effects of electron-electron interaction and charging energy are not taken into account in such a consideration. However, they might be important, for example, in small quasi-one-dimensional (quasi-1D) rings or in arrays of such rings fabricated in very recent experiments4.5.6.

In the present paper we calculate the linear tunneling conductance of the quasi-1D two-terminal ballistic ring with Rashba SO coupling threaded by a magnetic flux. The setup is schematically shown in Fig. 10 The spectrum of electrons in the ring is SO-split into two subbands. We will assume electron densities at which only the lowest radial band is occupied. The electron-electron interaction inside the ring is modeled by the parameters of the Tomonaga-Luttinger liquid (TLL), the leads being noninteracting. Assuming a weak tunneling between the leads and the ring, we compute the leading term of the Kubo conductance perturbatively expanded in a series of tunneling elements. We mostly follow the approach of Ref. 16 where a similar problem for spinless fermions was considered. We also make use of the bosonization in order to calculate the required TLL correlation functions. However, instead of the Matsubara formalism, we apply the Keldysh real-time approach to this quasiequilibrium problem (cf. Ref. 17). Such a combination of the Keldysh technique and bosonization appears more efficient for a derivation of asymptotic results at temperatures lower than the mean level spacing of the ring's spectrum.

After Ref. 18 it is known that an electron-electron interaction strongly renormalizes the height of tunneling barriers between the leads and TLL, and therefore at $T=$ 0 electron transport is suppressed. At finite temperatures $T \neq 0$ the linear conductance vanishes as a power law of $T$, while the effective width of a conductance peak grows with $T \rightarrow 0$. In order to ensure the validity of the weak-tunneling approximation, in our studies we assume a temperature range where the renormalized tunneling rates are smaller than the temperature, $\widetilde{\Gamma}_{l, r} \ll T$. On the other hand, finite-size effects remain important at $T \ll \omega_{0}$, the single-particle level spacing near the Fermi level.

In the temperature regime $\widetilde{\Gamma}_{l, r} \ll T \ll \omega_{0}$ the linear conductance is represented by a sequence of resonance peaks when plotted as a function of gate voltage and/or magnetic flux. In our paper we focus on the problem of how the distribution of the conductance peaks depends on the external parameters (magnetic flux, SO coupling, 


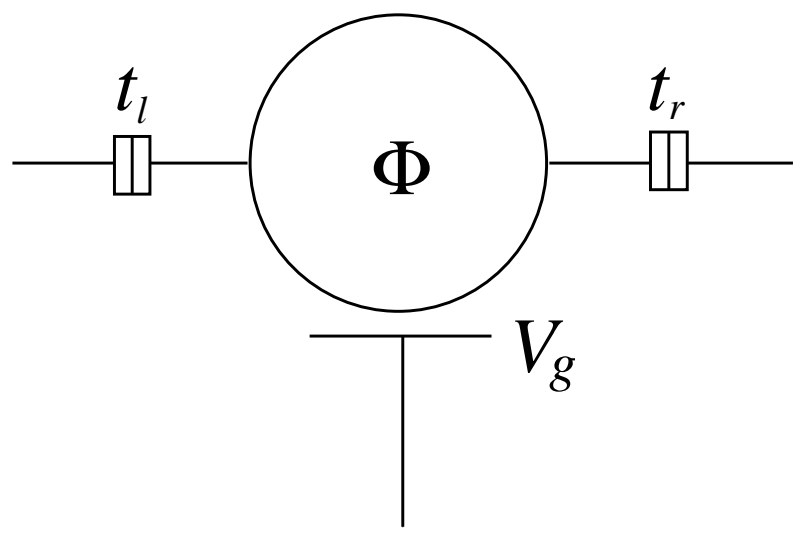

FIG. 1: The ring threaded by a magnetic flux $\Phi$ is weakly coupled to the leads through the tunneling barriers $t_{l}$ and $t_{r}$ and capacitively coupled to the gate electrode $\left(V_{g}\right)$.

gate voltage, charging energy) as well as on the parameters of the Tomonaga-Luttinger interaction. The perturbative expansion of the linear conductance in tunneling elements is known to break down in the resonance positions. Finding the poles of the leading term we can establish where the conductance peaks are centered. Thus, the study of electron transport in the TLL ring provides an effective tool of spectroscopy of its many-body states. Conceptually this is analogous to the study of the tunneling conductance between the two parallel quantum wires 19 which has been realized experimentally 20 . We note that a description of a shape of a particular peak is, however, a different problem which is usually tackled in a somewhat different manner (cf., e.g., Refs. 21 22 23), and it will not be addressed in the present context.

In our paper we extensively discuss the importance of the so-called Klein factors and zero modes (topological excitations) of the bosonized Hamiltonian ${ }^{24}$ for the description of distribution of conductance peaks. An accurate account of the Klein factors is necessary due to the presence of spin-orbit coupling in the system. The zero-mode sector of TLL decouples from its "continuous" (bosonic) sector and contains the whole dependence on external parameters $\frac{16.25}{}$. The latter appear in the topological sector after imposing boundary conditions. We elaborate on the procedure of averaging the conductance over zero modes in the presence of spin-orbit coupling and obtain analytically asymptotic results for the peak positions at temperatures lower than the mean level spacing. We also reexamine the case of spinless fermions reproducing the result of Ref. 16 and discuss it in further detail.

It is worthwhile to note that the relevance of the topological modes for a description of mesoscopic phenomena in the TLL rings has been already appreciated in various contexts, including studies of persistent ${ }^{26}$ and Josephson current $^{25}$ and the study of the AB phase in chiral Luttinger liquids 27 . The structure of the topological sector in the presence of SO coupling has been recently discussed as well in applications to persistent ${ }^{28,29}$ and Josephson ${ }^{30}$ currents.

The paper is organized as follows. In Sec. II we briefly outline the construction of the spectrum of the ring with SO coupling. In Sec. III we summarize the results emerging from an application of the Landauer-Büttiker formalism to this system. They will be further used as a reference in the noninteracting limit. In Sec. IV we present a derivation of the Kubo formula in the real-time approach. Briefly reviewing the bosonization formalism in Sec. V, we derive an expression for the dc conductance to be averaged over zero modes. The procedure of averaging is performed in Sec. VI. We discuss the interplay of the externally tuned and interaction parameters in the distribution of the conductance peaks, especially focusing on the modification of the Coulomb blockade due to SO coupling.

\section{MESOSCOPIC RINGS WITH RASHBA COUPLING: DISPERSION RELATIONS}

The two-dimensional electron gas with Rashba spinorbit coupling is described by the Hamiltonian

$$
H=\frac{1}{2 m^{*}}\left(p_{x}^{2}+p_{y}^{2}\right)+\alpha_{R}\left(\sigma_{x} p_{y}-\sigma_{y} p_{x}\right)+V(r),
$$

where $r=\sqrt{x^{2}+y^{2}}$. The magnetic field $\mathbf{B}$ is introduced in the kinetic momentum $\mathbf{p} \rightarrow \mathbf{p}+\frac{e}{c} \mathbf{A}$ via the gauge potential $\mathbf{A}=\frac{B}{2}(-y, x, 0)$. The radial potential $V(r)$ confining an electron to the ring geometry can be modeled, for example, either by singular isotropic harmonic oscillator or by concentric hard walls 29 . For these or any other types of the radial confinement the resulting quasi-one-dimensional spectrum $\varepsilon_{n \sigma}(k)$ is labeled by the radial band index $n=0,1, \ldots$, by the angular momentum $\hbar k=\ldots,-\hbar, 0, \hbar, \ldots$, and by the subband index (chirality) $\sigma= \pm$. From now on we will put $\hbar=1$.

If the effective ring's width is much smaller than the ring's radius, we can neglect the hybridization between the radial bands. We also assume electron densities at

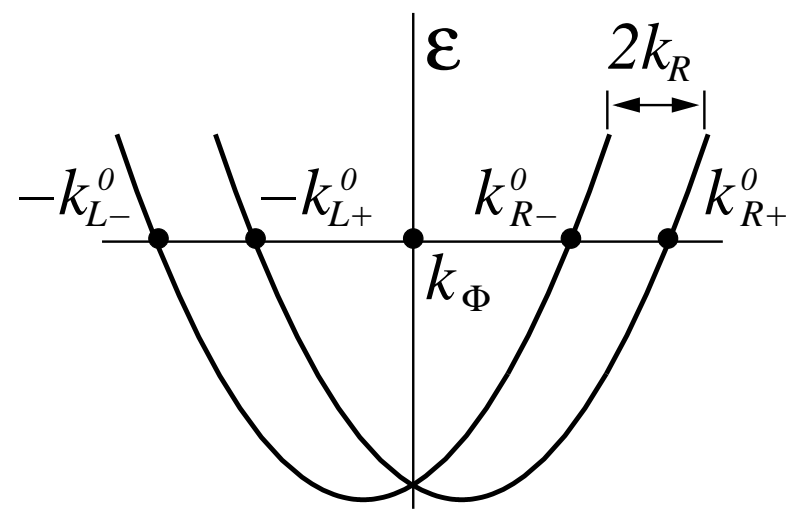

FIG. 2: The lowest radial band of the quasi-1D mesoscopic ring SO-split into two subbands. 
which only the lowest radial band $(n=0)$ is occupied. Thus, we effectively consider the strictly one-dimensional spectrum (see Fig. 2) which has a parabolic shape and is SO-split into two subbands 29 :

$$
\varepsilon_{\sigma}(k) \equiv \varepsilon_{0 \sigma}(k)=\frac{2 \pi^{2}}{m^{*} L^{2}}\left(k-k_{\Phi}-\sigma k_{R}\right)^{2} .
$$

Here $L$ is the ring's perimeter, $k_{\Phi}=\Phi / \Phi_{0}$ is a number of flux quanta $\Phi_{0}$ threading the ring, and the parameter

$$
k_{R}=\sqrt{\frac{1}{4}+\left(\frac{\alpha_{R} m^{*} L}{2 \pi}\right)^{2}}-\frac{1}{2}
$$

depends on the Rashba coupling $\alpha_{R}$.

Linearizing the spectrum (2) near the Fermi energy, we obtain the four branches

$$
\varepsilon_{\eta \sigma}(k)=\omega_{0}\left(k-k_{\eta \sigma}^{0}\right) \equiv \omega_{0}\left(k-\eta k_{F}-k_{\Phi}-\sigma k_{R}\right),
$$

specified by $\eta= \pm$ ( or $\eta=R, L$ ) and $\sigma= \pm$. The Fermi angular velocity $\omega_{0}=\left(\frac{2 \pi}{L}\right)^{2} \frac{k_{F}}{m^{*}}$ defines the level spacing of the spectrum (4), and $k_{F}$ is the Fermi angular momentum in absence of a magnetic field and SO coupling.

\section{CONDUCTANCE OF THE MESOSCOPIC RING: NONINTERACTING ELECTRONS}

Let us consider the conductance of the ring attached to the semi-infinite leads (Fig. 1). For noninteracting electrons it can be easily found in the framework of the scattering matrix theory 10 .

It is instructive to consider first the case of spinless fermions with the two linearization points $k_{R / L}^{0}$. One finds that in the zero-temperature limit and for the angle $\pi$ between the junctions to the leads the dc conductance reads 10

$$
G\left(k_{F}, k_{\Phi}\right)=\frac{e^{2}}{2 \pi} \frac{16 \epsilon_{l} \epsilon_{r} \sin ^{2} k_{F} \pi \cos ^{2} k_{\Phi} \pi}{\left[-2 \alpha_{l} \alpha_{r}+\left(1+\gamma_{l} \gamma_{r}\right) \cos 2 \pi k_{F}-2 \beta_{l} \beta_{r} \cos 2 \pi k_{\Phi}\right]^{2}+\left(1-\gamma_{l} \gamma_{r}\right)^{2} \sin ^{2} 2 \pi k_{F}}
$$

where $\epsilon_{l / r}, \gamma_{l / r}=-\sqrt{1-2 \epsilon_{l / r}}, \alpha_{l / r}=-\frac{1}{2}\left(1+\gamma_{l / r}\right)$, and $\beta_{l / r}=\frac{1}{2}\left(1-\gamma_{l / r}\right)$ are the phenomenological parameters describing scattering in a T-shaped (left $l$ or right $r$ ) junction. The number of flux quanta is given by $k_{\Phi}=\frac{1}{2}\left(k_{R}^{0}-k_{L}^{0}\right)$, while the quantity $k_{F}=\frac{1}{2}\left(k_{R}^{0}+k_{L}^{0}\right)$ corresponds to the Fermi momentum at zero flux. It can be replaced by $k_{F} \rightarrow N_{0}+\frac{\Delta \mu}{\omega_{0}}$, where $\Delta \mu$ is a difference between the chemical potential of the leads and the Fermi energy of the ring, and the integer $N_{0}$ is related to the number $2 N_{0}+1$ of electrons in the ring at $\Delta \mu=0$. Since the expression (5) is periodic in $k_{F}$, the integer part of $k_{F}$ can be discarded. Thus, the conductance (5) actually depends on the fractional part of $\frac{\Delta \mu}{\omega_{0}}$. For future references we introduce the parameter $k_{\mu}=\frac{\Delta \mu}{\omega_{0}}-\frac{1}{2}$.

In the weak-tunneling limit $\epsilon_{l / r} \stackrel{\omega_{0}}{\ll} 1$ the conductance (5) approximately equals

$$
G \approx \frac{e^{2}}{2 \pi} \frac{4 \epsilon_{l} \epsilon_{r} \sin ^{2} k_{F} \pi \cos ^{2} k_{\Phi} \pi}{\left(\cos 2 \pi k_{F}-\cos 2 \pi k_{\Phi}\right)^{2}+\frac{1}{4}\left(\epsilon_{l}+\epsilon_{r}\right)^{2} \sin ^{2} 2 \pi k_{F}} .
$$

As a function $k_{\mu}$ and $k_{\Phi}$, it represents a sequence of BreitWigner resonances. The conductance peaks occur when the resonance condition $\cos 2 \pi k_{F}=\cos 2 \pi k_{\Phi}$ is fulfilled - i.e., at the values of the parameters

$$
k_{F}+k_{\Phi}=n_{R}, \quad k_{F}-k_{\Phi}=n_{L}
$$

where $n_{R}$ and $n_{L}$ are arbitrary integers. We note that in the weak-tunneling limit the resonance condition (7) remains valid for arbitrary angle $x_{l}-x_{r}$ between the junctions, while the shape of Breit-Wigner resonances is quite sensitive to the value of $x_{l}-x_{r}$.

It has been demonstrated in Ref. 12 that for electrons with nonzero SO coupling and negligible Zeeman splitting the conductance of the mesoscopic ring is given by the sum of the two contributions: $G\left(k_{F}, k_{\Phi}+k_{R}\right)$ and $G\left(k_{F}, k_{\Phi}-k_{R}\right)$. In other words, the net effect of the SO coupling for noninteracting electrons is the generation of the different effective flux values for the different chan-

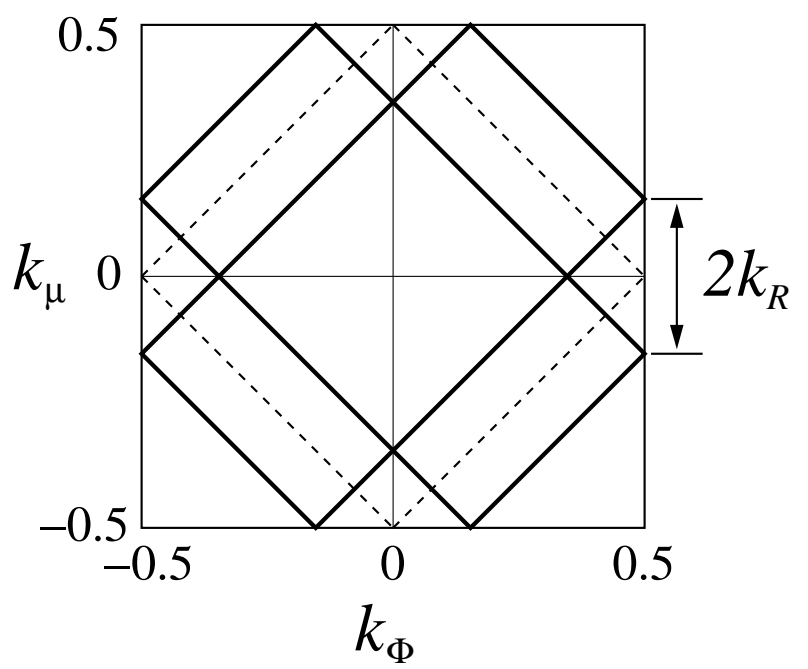

FIG. 3: Splitting of the conductance peaks (solid lines) due to SO coupling. The dashed lines correspond to $k_{R}=0$. 
nels. Therefore, the pattern of the conductance maxima at $k_{R} \neq 0$ is determined by the resonance conditions

$$
k_{F}+\left(k_{\Phi} \pm k_{R}\right)=n_{R \pm}, \quad k_{F}-\left(k_{\Phi} \pm k_{R}\right)=n_{L \pm},
$$

where $n_{R \pm}$ and $n_{L \pm}$ are arbitrary integers. Recalling that effectively $k_{\mu}=k_{F}-\frac{1}{2}$, we show in Fig. [3 how the arrangement of the conductance peaks is modified by SO coupling.

\section{KUBO FORMULA}

In order to take into account effects of the electronelectron interaction on the distribution of conductance peaks, we discuss in this section the Kubo formula for the linear conductance. Although this expression is very standard, we rederive it using the Keldysh formalism. In doing this, we pursue two objectives. First, we would like to have better control of the approximations used (similar to those made in Ref. 16). Second, we would like to deduce an expression for the conductance in a realtime representation. Its advantage for the ring geometry will be discussed in the next section where the calculation of time-dependent finite-size TLL correlation functions is concerned.

In the second-quantized formulation the mesoscopic ring attached to the leads is described by the Hamiltonian

$$
H=H_{l}+H_{r}+H_{c}+H_{T} .
$$

The left/right lead is described by a Fermi-liquid Hamiltonian $H_{l / r}=\int d x c_{l / r}^{\dagger}(x)\left(\frac{p^{2}}{2 m^{*}}-\mu\right) c_{l / r}(x)$, and the tunneling term is $H_{T}=\sum_{l, r}\left[t_{l / r} c_{l / r}^{\dagger}\left(x_{l / r}\right) \psi\left(x_{l / r}\right)+\right.$ H.c. $]$.
Here $c_{l / r}$ and $\psi$ are the field operators in the leads and in the ring, respectively.

The Hamiltonian of the central part (ring) $H_{c}\left[\psi^{\dagger}, \psi\right]$ can have any interaction term in addition to the kinetic term. In our consideration we will model the electronelectron interaction in the ring by the TomonagaLuttinger liquid which includes only forward-scattering processes ("density-density"-type interaction). In the framework of this model it is also possible to take into account the charging effects. They originate from a capacitive coupling of the ring to the gate electrode and are described by the Hamiltonian $E_{c}\left(N_{\text {ring }}-\frac{1}{e} C_{g} V_{g}\right)^{2}$, with the charging energy $E_{c}=e^{2} / 2 C_{g}$. Here $C_{g}$ is the gate capacitance and $\hat{N}_{\text {ring }}$ is the number operator of electrons in the ring.

The linear response of the system to an applied timedependent bias voltage is described by the Kubo formula for the ac conductance $\mathrm{e}^{31}$ :

$$
G(\Omega)=-\frac{1}{\Omega} \int_{-\infty}^{t} d t^{\prime} e^{-i \Omega\left(t^{\prime}-t\right)}\left\langle\left[\hat{I}_{l}(t), \hat{I}_{r}\left(t^{\prime}\right)\right]\right\rangle,
$$

where $\hat{I}_{l / r}(t)=i e\left[t_{l / r} \hat{c}_{l / r}^{\dagger}\left(x_{l / r}, t\right) \hat{\psi}\left(x_{l / r}, t\right)-\right.$ H.c. $]$ is a current operator in the left/right junction written in the Heisenberg representation.

In the weak-tunneling limit the expression (10) can be expanded in a series of $H_{T}$. We make use of the realtime Keldysh diagrammatic technique, and for $t>t^{\prime}$ we replace $\left\langle\left[\hat{I}_{l}(t), \hat{I}_{r}\left(t^{\prime}\right)\right]\right\rangle$ by

$$
\left\langle T_{t} \hat{I}_{l}(t) \hat{I}_{r}\left(t^{\prime}\right)\right\rangle-\left\langle\tilde{T}_{t} \hat{I}_{l}(t) \hat{I}_{r}\left(t^{\prime}\right)\right\rangle \equiv 2 i \operatorname{Im}\left\langle T_{t} \hat{I}_{l}(t) \hat{I}_{r}\left(t^{\prime}\right)\right\rangle .
$$

When expressed on the Keldysh contour, it reads

$$
\left\langle T_{t} \hat{I}_{l}(t), \hat{I}_{r}\left(t^{\prime}\right)\right\rangle=\left\langle\tilde{T}_{t} e^{i \int_{-\infty}^{t} H_{T}\left(t^{\prime \prime}\right) d t^{\prime \prime}} I_{l}(t) T_{t} e^{-i \int_{-\infty}^{t} H_{T}\left(t^{\prime \prime}\right) d t^{\prime \prime}} \tilde{T}_{t} e^{i \int_{-\infty}^{t^{\prime \prime}} H_{T}\left(t^{\prime \prime}\right) d t^{\prime \prime}} I_{r}\left(t^{\prime}\right) T_{t} e^{-i \int_{-\infty}^{t^{\prime}} H_{T}\left(t^{\prime \prime}\right) d t^{\prime \prime}}\right\rangle
$$

where the operators without carets refer to the interaction $\left(H_{T}\right)$ representation.

Expanding (11) to the second order in $H_{T}$, we obtain

$$
\begin{aligned}
& \int_{-\infty}^{t} d t_{1} \int_{-\infty}^{t^{\prime}} d t_{1}^{\prime}\left\langle\left[\left[I_{l}(t), H_{T}\left(t_{1}\right)\right],\left[H_{T}\left(t_{1}^{\prime}\right), I_{r}\left(t^{\prime}\right)\right]\right]\right\rangle \\
- & \int_{-\infty}^{t} d t_{1} \int_{-\infty}^{t_{1}} d t_{2}\left\langle\left[\left[\left[I_{l}(t), H_{T}\left(t_{1}\right)\right], H_{T}\left(t_{2}\right)\right], I_{r}\left(t^{\prime}\right)\right]\right\rangle \\
- & \int_{-\infty}^{t^{\prime}} d t_{1}^{\prime} \int_{-\infty}^{t_{1}^{\prime}} d t_{2}^{\prime}\left\langle\left[I_{l}(t),\left[H_{T}\left(t_{2}^{\prime}\right),\left[H_{T}\left(t_{1}^{\prime}\right), I_{r}\left(t^{\prime}\right)\right]\right]\right]\right\rangle .
\end{aligned}
$$

The next step is to perform averaging over the leads' states. While doing this, we meet the following combinations: (a) $\left(G_{l}^{R}-G_{l}^{A}\right)\left(G_{r}^{R}-G_{r}^{A}\right)$, (b) $G_{l}^{K}\left(G_{r}^{R}-G_{r}^{A}\right)$, (c) $\left(G_{l}^{R}-G_{l}^{A}\right) G_{r}^{K}$, and (d) $G_{l}^{K} G_{r}^{K}$. Here $G_{l / r}^{R, A}$ and $G_{l / r}^{K}$ are the momentum-averaged retarded, advanced, and Keldysh functions of the leads in the real-time representation

$$
\begin{array}{r}
\left(G^{R}-G^{A}\right)_{l / r}(t)=-i\left\langle\left\{c_{l / r}(t), c_{l / r}^{\dagger}(0)\right\}\right\rangle=-2 \pi i \delta(t) \frac{\nu_{l / r}}{V_{l / r}} \\
G_{l / r}^{K}(t)=-i\left\langle\left[c_{l / r}(t), c_{l / r}^{\dagger}(0)\right]\right\rangle=-\frac{2 \pi}{\beta \sinh (\pi t / \beta)} \frac{\nu_{l / r}}{V_{l / r}},
\end{array}
$$

and $\nu_{l / r}$ is the density of states in the left/right lead at the Fermi level.

One can straightforwardly prove that the combinations (a) and (b) vanish identically. The combination (c) gives 
the following contribution to the conductance:

$$
\begin{aligned}
G^{(c)}(\Omega)= & e^{2} \Gamma_{l} \Gamma_{r} L^{2} \int_{-\infty}^{0} \int_{-\infty}^{0} d t_{1} d t_{2} \frac{e^{-i \Omega t_{1}}\left(1-e^{-i \Omega t_{2}}\right)}{2 i \Omega \beta \sinh \left[\pi t_{2} / \beta\right]} \\
& \times \operatorname{Re}\left\langle\left\{\left[\left[\psi_{l}(0), \psi_{l}^{\dagger}(0)\right], \psi_{r}\left(t_{1}\right)\right], \psi_{r}^{\dagger}\left(t_{1}+t_{2}\right)\right\}\right\rangle, \quad(13)
\end{aligned}
$$

where $\Gamma_{l / r}=2 \pi \nu_{l / r}\left|t_{l / r}\right|^{2} /\left(V_{l / r} L\right)$ and $V_{l / r}$ is the volume of the left/right lead.

From Eq. (13) we derive an expression for the dc conductance $(\Omega=0)$ at zero temperature

$$
\begin{aligned}
G^{(c)} & =\frac{e^{2}}{2 \pi} \Gamma_{l} \Gamma_{r} L^{2} \int_{-\infty}^{0} \int_{-\infty}^{0} d t_{1} d t_{2} \\
& \times \operatorname{Re}\left\langle\left\{\left[\left[\psi_{l}(0), \psi_{l}^{\dagger}(0)\right], \psi_{r}\left(t_{1}\right)\right], \psi_{r}^{\dagger}\left(t_{1}+t_{2}\right)\right\}\right\rangle
\end{aligned}
$$

Using the operator identities

$$
\begin{array}{r}
\{[C, A], B\}+\{[C, B], A\}=[C,\{A, B\}], \\
\{C,\{A, B\}\}-\{A,\{C, B\}\}=[[C, A], B],
\end{array}
$$

we rewrite Eq. (14),

$$
\begin{aligned}
G^{(c)}= & \frac{e^{2}}{4 \pi} \Gamma_{l} \Gamma_{r} L^{2} \int_{-\infty}^{0} \int_{-\infty}^{0} d t_{1} d t_{2} \\
\times & \left\{\operatorname{Re}\left\langle\left\{\left[\left[\psi_{l}(0), \psi_{l}^{\dagger}(0)\right], \psi_{r}\left(t_{1}\right)\right], \psi_{r}^{\dagger}\left(t_{2}\right)\right\}\right\rangle\right. \\
& \left.+\operatorname{Re}\left\langle\left[\left[\psi_{l}(0), \psi_{l}^{\dagger}(0)\right],\left\{\psi_{r}\left(t_{1}\right), \psi_{r}^{\dagger}\left(t_{2}\right)\right\}\right]\right\rangle\right\},
\end{aligned}
$$

and further express

$$
\begin{aligned}
& \left\langle\left\{\left[\left[\psi_{l}(0), \psi_{l}^{\dagger}(0)\right], \psi_{r}\left(t_{1}\right)\right], \psi_{r}^{\dagger}\left(t_{2}\right)\right\}\right\rangle \\
= & \left\langle\left\{\left\{\psi_{l}(0), \psi_{r}^{\dagger}\left(t_{2}\right)\right\},\left\{\psi_{r}\left(t_{1}\right), \psi_{l}^{\dagger}(0)\right\}\right\}\right\rangle \\
+ & \left\langle\left[\left[\psi_{r}^{\dagger}\left(t_{2}\right),\left\{\psi_{l}^{\dagger}(0), \psi_{r}\left(t_{1}\right)\right\}\right], \psi_{l}(0)\right]\right\rangle \\
- & \left\langle\left\{\left\{\psi_{l}^{\dagger}(0),\left\{\psi_{l}(0), \psi_{r}\left(t_{1}\right)\right\}\right\}, \psi_{r}^{\dagger}\left(t_{2}\right)\right\}\right\rangle .
\end{aligned}
$$

It is obvious that in the noninteracting limit the only term $\left\langle\left\{\left\{\psi_{l}(0), \psi_{r}^{\dagger}\left(t_{2}\right)\right\},\left\{\psi_{r}\left(t_{1}\right), \psi_{l}^{\dagger}(0)\right\}\right\}\right\rangle$ survives, since the other terms vanish due to the fermionic commutation relations. We approximate the dc conductance in the interacting case by this dominant contribution

$$
\begin{aligned}
G & \approx \frac{e^{2}}{4 \pi} \Gamma_{l} \Gamma_{r} L^{2} \int_{-\infty}^{0} \int_{-\infty}^{0} d t_{1} d t_{2} \\
& \times \operatorname{Re}\left\langle\left\{\left\{\psi_{l}(0), \psi_{r}^{\dagger}\left(t_{2}\right)\right\},\left\{\psi_{r}\left(t_{1}\right), \psi_{l}^{\dagger}(0)\right\}\right\}\right\rangle .
\end{aligned}
$$

Splitting the four-particle correlator, one can recover the formula $G \approx\left(e^{2} / 2 \pi\right) \Gamma_{l} \Gamma_{r} L^{2}\left|G^{R}\left(\omega=0, x_{l}-x_{r}\right)\right|^{2}$ from Ref. 16, where $G^{R}\left(\omega=0, x_{l}-x_{r}\right)$ is a zero-frequency retarded Green's function for interacting electrons in the ring. This approximation physically means that one scattering event is completed before another takes place. In general, the TLL correlation functions of any order can be calculated within the bosonization approach, and this approximation can be relaxed.

The combination (d) with $G_{l}^{K} G_{r}^{K}$ also gives a finite contribution to the conductance, which, however, vanishes in the noninteracting limit as well. Therefore, we will neglect it on the same ground as we have just neglected the subdominant terms in Eqs. (17) and (18).

\section{BOSONIZATION}

\section{A. Spinless case}

In order to compute the four-particle correlator (19), we will make use of the bosonization technique 24 .

Let us consider for simplicity the spinless case. We introduce the shorthand notations for the fermionic fields $\psi_{l} \equiv \psi\left(x_{l}\right)$ and $\psi_{r} \equiv \psi\left(x_{r}\right)$, where $x_{l}$ and $x_{r}$ are the angle coordinates of the left and right junctions. In the following we assume that $x_{l}=0$ and $x_{r}=\pi$.

In the bosonization the fields $\psi_{l / r}$ are represented as a sum of the right- $(\eta=+$, or $R)$ and left- $(\eta=-$, or $L)$ moving components,

$$
\psi_{l / r}=\psi_{l / r, R}+\psi_{l / r, L}=F_{l / r, R} \psi_{l / r, R}^{b}+F_{l / r, L} \psi_{l / r, L}^{b},
$$

and each of $\psi_{l / r, \eta}$ consists of a topological part $F_{l / r, \eta}$ and a bosonic part $\psi_{l / r, \eta}^{b}$ commuting with each other: $\left[F, \psi^{b}\right]=0$.

The bosonic part is given by

$$
\begin{aligned}
& \psi_{\eta}^{b}(x)=\frac{1}{\sqrt{L \tilde{\alpha}}} e^{-i \sqrt{2 \pi} \phi_{\eta}(x)}, \\
& \phi_{\eta}(x)=i \sum_{k=1}^{\infty} \frac{e^{-\frac{1}{2} \tilde{\alpha} k}}{\sqrt{2 \pi k}}\left(e^{i \eta k x} b_{\eta k}-e^{-i \eta k x} b_{\eta k}^{\dagger}\right),
\end{aligned}
$$

where $\tilde{\alpha}=\frac{2 \pi \alpha}{L}$ is a small dimensionless cutoff parameter and the operators $b_{\eta k}, b_{\eta k}^{\dagger}$ satisfy the bosonic commutation relation $\left[b_{\eta k}, b_{\eta^{\prime} k^{\prime}}^{\dagger}\right]=\delta_{\eta \eta^{\prime}} \delta_{k k^{\prime}}$.

The topological part is important for the finite-size TLL with periodic boundary conditions. It includes Klein factors $F_{\eta}$, zero-mode operators $N_{\eta}$, and the linearization points $k_{\eta}^{0}$ (see Fig. [2] assuming $k_{R}=0$ ):

$$
F_{l / r, \eta}=F_{\eta} e^{i \eta\left(N_{\eta}-k_{\eta}^{0}\right) x_{l / r}} .
$$

The zero-mode operators $N_{\eta}=N_{\eta}^{\dagger}$ take integer values, and the following relations are satisfied ${ }^{24}$ :

$$
\begin{aligned}
{\left[F_{\eta}, N_{\eta^{\prime}}\right] } & =F_{\eta} \delta_{\eta \eta^{\prime}}, \\
\left\{F_{\eta}, F_{\eta^{\prime}}^{\dagger}\right\} & =2 \delta_{\eta \eta^{\prime}}, \\
\left\{F_{\eta}, F_{\eta^{\prime}}\right\} & =\left\{F_{\eta}^{\dagger}, F_{\eta^{\prime}}^{\dagger}\right\}=0 \text { for } \eta \neq \eta^{\prime} .
\end{aligned}
$$

The bosonized TLL Hamiltonian $H_{T L L} \equiv H_{c}=H_{b}+$ $H_{0}$ consists of a "continuous" (bosonic) $H_{b}$ part and a topological $H_{0}$ part which are decoupled from each other. Therefore, the factorization of $\psi_{l / r, \eta}$ into $F_{l / r, \eta}$ and $\psi_{l / r, \eta}^{b}$ takes place at any time instant:

$$
\psi_{l / r}(t)=F_{l / r, R}(t) \psi_{l / r, R}^{b}(t)+F_{l / r, L}(t) \psi_{l / r, L}^{b}(t),
$$

where the time evolutions of $\psi_{l / r, \eta}^{b}(t)$ and $F_{l / r, \eta}(t)$ are governed by $H_{b}$ and $H_{0}$, respectively. By the same reason the statistical averagings in both bosonic and topological sectors are independent of each other. 
The bosonic part of the TLL Hamiltonian is given by

$$
H_{b}=\frac{2 \pi v}{L} \sum_{a=1,2} \sum_{k=1}^{\infty} k d_{a k}^{\dagger} d_{a k}
$$

where $v$ is the so-called charge velocity (the renormalization of the Fermi velocity $\left.v_{0} \equiv \frac{L \omega_{0}}{2 \pi}\right)$. The operators $d_{a k}$, $d_{a k}^{\dagger}(a=1,2)$ are obtained from $b_{\eta k}, b_{\eta k}^{\dagger}$ by the canonical transformation (A1). The latter depends on the interaction parameter $\gamma=\frac{1}{2}\left(\frac{1}{K}+K\right)$, where $K$ is the so-called charge stiffness. For repulsive interactions $K<1$, while in the noninteracting limit $K=\gamma=1$ and $v=v_{0}$.

The topological part of the TLL Hamiltonian is

$$
H_{0}=\sum_{\eta}\left(a_{0} \tilde{N}_{\eta}^{2}+a_{1} \tilde{N}_{\eta} \tilde{N}_{-\eta}\right)
$$

where $a_{0,1}=\frac{\omega_{0}}{4}(\tilde{\nu} \pm \lambda)$ and

$$
\tilde{\nu}=\nu+\frac{4 E_{c}}{\omega_{0}}, \quad \nu=\frac{v}{K v_{0}}, \quad \lambda=\frac{v K}{v_{0}} .
$$

The topological numbers $\tilde{N}_{\eta}=N_{\eta}-k_{\eta}$ are shifted by $k_{\eta}=k_{\eta}^{0}+\delta k_{\mu}$, where

$$
\delta k_{\mu}=\frac{4 E_{c}\left(\frac{1}{e} C_{g} V_{g}-2 N_{0}\right)+2 \Delta \mu-\omega_{0}}{2 \tilde{\nu} \omega_{0}}
$$

redefines the linearization points $k_{\eta}^{0}$ in order to include the dependence on $\Delta \mu$ and the gate voltage $V_{g}$. In the basis $N=N_{R}+N_{L}, J=N_{R}-N_{L}$, the Hamiltonian $H_{0}$ acquires the diagonal form

$$
H_{0}=\frac{\omega_{0}}{4}\left[\tilde{\nu} \tilde{N}^{2}+\lambda \tilde{J}^{2}\right]
$$

where $\tilde{N}=N-2 k_{\mu}, \tilde{J}=J-2 k_{\Phi}$, and $k_{\mu}=N_{0}+\delta k_{\mu}$. One can observe that the whole dependence on $\Delta \mu, V_{g}$, and $\Phi$ is included in the topological sector.

Using the commutation relations (23) we find the time evolution of the Klein factors

$$
F_{\eta}(t)=e^{i H_{0} t} F_{\eta} e^{-i H_{0} t}=F_{\eta} e^{-i t P_{\eta}+i t a_{0}},
$$

where

$$
P_{\eta}=2 a_{0} \tilde{N}_{\eta}+2 a_{1} \tilde{N}_{-\eta}=\frac{\omega_{0}}{2}[\tilde{\nu} \tilde{N} \pm \lambda \tilde{J}] .
$$

The details of the time evolution of the bosonic fields are presented in Appendix $\mathrm{A}$ In fact, they are not very important for our purpose. We will only exploit the fact that the average of the bosonic fields,

$$
g^{b}(t ; \gamma)=\left\langle\psi_{l R}^{b}(t) \psi_{r R}^{b \dagger}(0)\right\rangle \equiv\left\langle\psi_{l L}^{b}(t) \psi_{r L}^{b \dagger}(0)\right\rangle,
$$

is a periodic function of time which can be expanded in a Fourier series

$$
g^{b}(t ; \gamma)=\sum_{p=0}^{\infty} g_{p}(\gamma) e^{-i p \omega t},
$$

with frequency $\omega=\frac{2 \pi v}{L}$ and real-valued coefficients $g_{p}(\gamma)$. Note that the summation in Eq. [35] is performed only over non-negative integers.

The real-time periodicity of $g^{b}(t ; \gamma)$ is inherited from the spatial periodic boundary conditions. The occurrence of the Fourier series (35) allows us to perform all time integrals explicitly. The analysis of the remaining series is a much simpler task.

Let us make yet another approximation in the spirit of Ref. 16. In particular, we split the four-particle bosonic correlator in (19), neglecting the anomalous averages (e.g., $\left.\left\langle\psi^{b} \psi^{b}\right\rangle\right)$, the left-right mixing (e.g., $\left\langle\psi_{L}^{b} \psi_{R}^{b \dagger}\right\rangle$ ), and the vertex corrections (averages of operators at the same spatial point, e.g., $\left.\left\langle\psi_{r}^{b} \psi_{r}^{b \dagger}\right\rangle\right)$ in the bosonic (continuous) sector. At the same time, we do not split the topological part of the four-particle correlator (unlike has been done in Ref. 16) and perform a single averaging of the whole over zero modes.

Implementing this procedure, we obtain

$$
\begin{aligned}
& \left\langle\psi_{l}(0) \psi_{r}^{\dagger}\left(t_{2}\right) \psi_{r}\left(t_{1}\right) \psi_{l}^{\dagger}(0)\right\rangle \approx g^{b *}\left(t_{2}\right) g^{b}\left(t_{1}\right) \\
& \times \sum_{\eta_{1}, \eta_{2}}\left\langle F_{l, \eta_{2}}(0) F_{r, \eta_{2}}^{\dagger}\left(t_{2}\right) F_{r, \eta_{1}}\left(t_{1}\right) F_{l, \eta_{1}}^{\dagger}(0)\right\rangle_{z . m .} .
\end{aligned}
$$

where $\langle\cdots\rangle_{z . m}$. implies averaging over zero modes to be discussed later. Collecting all contributions, we find

$$
\begin{aligned}
G \approx & \frac{e^{2}}{2 \pi} \Gamma_{l} \Gamma_{r} L^{2} \sum_{p_{1}, p_{2}=0}^{\infty} g_{p_{1}}(\gamma) g_{p_{2}}(\gamma) \int_{-\infty}^{0} \int_{-\infty}^{0} d t_{1} d t_{2} \\
\times & \sum_{\eta= \pm} \operatorname{Re}\left\langle\left(e^{i t_{1}\left(\omega p_{1}+a_{0}+P_{\eta}\right)}-e^{-i t_{1}\left(\omega p_{1}+a_{0}-P_{\eta}\right)}\right)\right. \\
& \times\left(e^{-i t_{2}\left(\omega p_{2}+a_{0}+P_{\eta}\right)}-e^{i t_{2}\left(\omega p_{2}+a_{0}-P_{\eta}\right)}\right) \\
+ & e^{i\left(N_{\eta}+N_{-\eta}\right) \pi}\left(e^{i \eta t_{1}\left(\omega p_{1}+a_{0}+P_{\eta}\right)}-e^{-i \eta t_{1}\left(\omega p_{1}+a_{0}-P_{\eta}\right)}\right) \\
& \left.\times\left(e^{-i \eta t_{2}\left(\omega p_{2}+a_{0}+P_{-\eta}\right)}-e^{i \eta t_{2}\left(\omega p_{2}+a_{0}-P_{-\eta}\right)}\right)\right\rangle_{z . m .} .
\end{aligned}
$$

Introducing

$$
A_{\eta}^{ \pm}=\sum_{p=0}^{\infty} \frac{g_{p}(\gamma)}{\omega p+a_{0} \pm P_{\eta}}
$$

and $A_{\eta}=A_{\eta}^{+}+A_{\eta}^{-}$, we can cast Eq. (37) into the form

$$
\begin{aligned}
G & \approx \frac{e^{2}}{2 \pi} \Gamma_{l} \Gamma_{r} L^{2}\left\langle A_{R}^{2}+A_{L}^{2}\right. \\
& \left.+2 A_{R} A_{L} \cos \left(\tilde{N}_{R}+\tilde{N}_{L}+2 \delta k_{\mu}\right) \pi\right\rangle_{z . m .} .
\end{aligned}
$$

We remark that the alteration of the angle $x_{l}-x_{r}$ between the junctions would only modify the Fourier coefficients $g_{p}(\gamma)$ in Eq. (38) as well as the relative phase of the interference term $\sim A_{R} A_{L}$ in Eq. (39). Meanwhile, the poles of $A_{\eta}^{ \pm}$in Eq. (38) are not sensitive to the value of $x_{l}-x_{r}$.

In order to treat further the expression (39) we need to establish an efficient procedure of averaging over zero 
modes. But first we are going to discuss the modification of the conductance (39) caused by the presence of spin degrees of freedom and by spin-orbit coupling.

\section{B. Spinful case}

Performing a similar bosonization procedure in the spinful case, we obtain the following expression for the dc conductance

$$
\begin{aligned}
G & \approx \frac{e^{2}}{2 \pi} \Gamma_{l} \Gamma_{r} L^{2} \sum_{\sigma= \pm}\left\langle A_{R \sigma}^{2}+A_{L \sigma}^{2}\right. \\
& \left.+2 A_{R \sigma} A_{L \sigma} \cos \left(\tilde{N}_{R \sigma}+\tilde{N}_{L \sigma}+2 \delta k_{\mu}\right) \pi\right\rangle_{z . m .}
\end{aligned}
$$

The zero-mode operators $N_{\eta \sigma}$ with integer eigenvalues are shifted to $\tilde{N}_{\eta \sigma}=N_{\eta \sigma}-k_{\eta \sigma}$ by $k_{\eta \sigma}=k_{\eta \sigma}^{0}+\delta k_{\mu}$, where

$$
\delta k_{\mu}=\frac{4 E_{c}\left(\frac{1}{e} C_{g} V_{g}-4 N_{0}\right)+2 \Delta \mu-\omega_{0}}{2 \tilde{\nu}_{c} \omega_{0}} .
$$

The integer $N_{0}=\frac{1}{4} \sum_{\eta, \sigma} k_{\eta \sigma}^{0}$ is related to the number $4 N_{0}+2$ of electrons in the ring when the parameters

$$
\begin{aligned}
k_{\Phi} & =\frac{1}{4} \sum_{\sigma}\left(k_{R \sigma}-k_{L \sigma}\right), \\
k_{B, R} & =\frac{1}{4} \sum_{\sigma} \sigma\left(k_{R \sigma} \pm k_{L \sigma}\right)
\end{aligned}
$$

equal zero. The parameter $k_{B}$ vanishes in the absence of a Zeeman interaction. The parameter

$$
k_{\mu}=\frac{1}{4} \sum_{\eta, \sigma} k_{\eta \sigma}=N_{0}+\delta k_{\mu}
$$

contains the dependence on $\Delta \mu$ and $V_{g}$.

Like in the spinless case, it is convenient to introduce

$$
\tilde{\nu}_{c}=\nu_{c}+\frac{8 E_{c}}{\omega_{0}}, \quad \nu_{c, s}=\frac{v_{c, s}}{K_{c, s} v_{0}}, \quad \lambda_{c, s}=\frac{v_{c, s} K_{c, s}}{v_{0}}
$$

and $\omega_{c, s}=\frac{2 \pi v_{c, s}}{L}$, and $\gamma_{c, s}=\frac{1}{2}\left(\frac{1}{K_{c, s}}+K_{c, s}\right)$, which are expressed through the charge and spin velocities $v_{c} \neq v_{s}$, the charge and spin stiffnesses $K_{c} \neq K_{s}$, and the charging energy $E_{c}$.

In Eq. (40) the rates $\Gamma_{l}$ and $\Gamma_{r}$ remain the same as in the spinless case, since we assume that the density of states in the leads is spin independent and equals $\nu_{l / r}$ for each spin component. The spin dependence appears in the functions $A_{\eta \sigma}=A_{\eta \sigma}^{+}+A_{\eta \sigma}^{-}$,

$$
\begin{aligned}
A_{\eta \sigma}^{ \pm} & =\sum_{p_{c}, p_{s}=0}^{\infty} \frac{g_{p_{c}}\left(\frac{1}{2} \gamma_{c}\right) g_{p_{s}}\left(\frac{1}{2} \gamma_{s}\right)}{\omega_{c} p_{c}+\omega_{s} p_{s}+\bar{a}_{0} \pm P_{\eta \sigma}} \\
P_{\eta \sigma} & =2 \bar{a}_{0} \tilde{N}_{\eta \sigma}+2 \bar{a}_{1} \tilde{N}_{-\eta, \sigma} \\
& +2 \bar{a}_{2} \tilde{N}_{\eta,-\sigma}+2 \bar{a}_{3} \tilde{N}_{-\eta,-\sigma} .
\end{aligned}
$$

The coefficients $\bar{a}_{0,1}=\frac{\omega_{0}}{8}\left(\tilde{\nu}_{c} \pm \lambda_{c}+\nu_{s} \pm \lambda_{s}\right)$ and $\bar{a}_{2,3}=$ $\frac{\omega_{0}}{8}\left(\tilde{\nu}_{c} \pm \lambda_{c}-\nu_{s} \mp \lambda_{s}\right)$ are the components of the quadratic form of the zero-mode Hamiltonian

$$
\begin{aligned}
H_{0} & =\sum_{\eta, \sigma}\left(\bar{a}_{0} \tilde{N}_{\eta \sigma}^{2}+\bar{a}_{1} \tilde{N}_{\eta \sigma} \tilde{N}_{-\eta, \sigma}\right. \\
& \left.+\bar{a}_{2} \tilde{N}_{\eta \sigma} \tilde{N}_{\eta,-\sigma}+\bar{a}_{3} \tilde{N}_{\eta \sigma} \tilde{N}_{-\eta,-\sigma}\right)
\end{aligned}
$$

In the basis

$$
\begin{aligned}
N_{c, s} & =\left(N_{R+}+N_{L+}\right)+\sigma\left(N_{R-}+N_{L-}\right), \\
J_{c, s} & =\left(N_{R+}-N_{L+}\right)+\sigma\left(N_{R-}-N_{L-}\right),
\end{aligned}
$$

the Hamiltonian (48) becomes diagonal:

$$
H_{0}=\frac{\omega_{0}}{8}\left[\tilde{\nu}_{c} \tilde{N}_{c}^{2}+\lambda_{c} \tilde{J}_{c}^{2}+\nu_{s} \tilde{N}_{s}^{2}+\lambda_{s} \tilde{J}_{s}^{2}\right]
$$

and

$$
P_{\eta \sigma}=\frac{\omega_{0}}{4}\left[\left(\tilde{\nu}_{c} \tilde{N}_{c}+\eta \lambda_{c} \tilde{J}_{c}\right)+\sigma\left(\nu_{s} \tilde{N}_{s}+\eta \lambda_{s} \tilde{J}_{s}\right)\right],
$$

where $\tilde{N}_{c, s}=N_{c, s}-4 k_{\mu, B}$ and $\tilde{J}_{c, s}=J_{c, s}-4 k_{\Phi, R}$.

In Eq. (40) the two components $\sigma= \pm$ seem to be independent of each other. However, this is not the case, and they are, in fact, entangled due to the nontrivial procedure of averaging over zero modes.

\section{AVERAGING OVER ZERO MODES}

\section{A. Spinless case}

The typical expression to be averaged over zero modes before the time integration has the form [cf. Eq. (37)]

$$
\left\langle e^{i b_{1} \tilde{N}+i b_{2} \tilde{J}}\right\rangle_{z . m .}=\frac{\operatorname{Tr}\left(e^{i b_{1} \tilde{N}+i b_{2} \tilde{J}} e^{-\beta H_{0}}\right)}{\operatorname{Tr}\left(e^{-\beta H_{0}}\right)},
$$

where $b_{1,2}$ depend linearly on the time arguments $t_{1,2}$. The trace operation is understood as a summation over all possible integer values of $N_{L}$ and $N_{R}$. In the basis $(N, J)$ we have to sum over either both even $(2 m, 2 n)$ or both odd $(2 m+1,2 n+1)$ eigenvalues. Thus,

$$
\begin{gathered}
\operatorname{Tr}\left(e^{-\beta H_{0}}\right)=\sum_{m, n=-\infty}^{\infty} e^{-\beta \omega_{0}\left[\tilde{\nu}\left(m-k_{\mu}\right)^{2}+\lambda\left(n-k_{\Phi}\right)^{2}\right]} \\
+\sum_{m, n=-\infty}^{\infty} e^{-\beta \omega_{0}\left[\tilde{\nu}\left(m+\frac{1}{2}-k_{\mu}\right)^{2}+\lambda\left(n+\frac{1}{2}-k_{\Phi}\right)^{2}\right]} \\
=\frac{\pi}{\beta \omega_{0} \sqrt{\tilde{\nu} \lambda}}\left[\theta_{3}\left(\pi k_{\mu}, e^{-\frac{\pi^{2}}{\beta \omega_{0} \tilde{\nu}}}\right) \theta_{3}\left(\pi k_{\Phi}, e^{-\frac{\pi^{2}}{\beta \omega_{0} \lambda}}\right)\right. \\
\left.+\theta_{4}\left(\pi k_{\mu}, e^{-\frac{\pi^{2}}{\beta \omega_{0} \tilde{\nu}}}\right) \theta_{4}\left(\pi k_{\Phi}, e^{-\frac{\pi^{2}}{\beta \omega_{0} \lambda}}\right)\right]
\end{gathered}
$$

where $\theta_{3,4}$ are the Jacobian theta functions. Some properties of these functions are reviewed in Appendix B] 
The numerator in Eq. (53) can be equivalently rewritten in the form

$$
\operatorname{Tr}\left(e^{i b_{1} \tilde{N}+i b_{2} \tilde{J}} e^{-\beta H_{0}}\right)=\operatorname{Tr}\left(e^{-\beta H_{0}^{\prime}}\right) \cdot e^{-\frac{1}{\beta \omega_{0}}\left(\frac{b_{1}^{2}}{\tilde{\nu}}+\frac{b_{2}^{2}}{\lambda}\right)},
$$

where $H_{0}^{\prime}$ is obtained from $H_{0}$ by replacing

$$
\begin{aligned}
& k_{\mu} \rightarrow k_{\mu}^{\prime}=k_{\mu}+\frac{i b_{1}}{\beta \omega_{0} \tilde{\nu}}, \\
& k_{\Phi} \rightarrow k_{\Phi}^{\prime}=k_{\Phi}+\frac{i b_{2}}{\beta \omega_{0} \lambda} .
\end{aligned}
$$

At low temperatures $\beta^{-1} \ll \omega_{0}, E_{c}$, the last exponential factor in Eq. (55) can be discarded. Using Eq. (B5) we derive the following expression:

$$
\begin{aligned}
& \left\langle e^{i b_{1} \tilde{N}+i b_{2} \tilde{J}}\right\rangle_{z . m .} \approx p_{1}\left(k_{\mu}, k_{\Phi}\right) e^{2 i b_{1} f\left(k_{\mu}\right)+2 i b_{2} f\left(k_{\Phi}\right)} \\
& \quad+p_{2}\left(k_{\mu}, k_{\Phi}\right) e^{2 i b_{1} f\left(k_{\mu}+1 / 2\right)+2 i b_{2} f\left(k_{\Phi}+1 / 2\right)}
\end{aligned}
$$

The "sawtooth" function

$$
f(x)=\sum_{n=1}^{\infty} \frac{(-1)^{n}}{\pi n} \sin 2 \pi n x
$$

has the period 1 and equals $f(x)=-x$ for $x \in\left(-\frac{1}{2}, \frac{1}{2}\right)$ and $f\left( \pm \frac{1}{2}\right)=0$. The functions $p_{1,2}\left(k_{\mu}, k_{\Phi}\right)$ are determined by

$$
\begin{aligned}
p_{1}\left(k_{\mu}, k_{\Phi}\right) & =\frac{1}{1+\frac{\theta_{4}\left(\pi k_{\mu}, e^{-\pi^{2} / \beta \omega_{0} \tilde{\nu}}\right) \theta_{4}\left(\pi k_{\Phi}, e^{-\pi^{2} / \beta \omega_{0} \lambda}\right)}{\theta_{3}\left(\pi k_{\mu}, e^{-\pi^{2} / \beta \omega_{0} \tilde{\nu}}\right) \theta_{3}\left(\pi k_{\Phi}, e^{-\pi^{2} / \beta \omega_{0} \lambda}\right)}} \\
& \approx \frac{1}{1+e^{\beta \omega_{0}\left[\tilde{\nu} g_{2}\left(k_{\mu}\right)+\lambda g_{2}\left(k_{\Phi}\right)\right]}},
\end{aligned}
$$

and $p_{2}\left(k_{\mu}, k_{\Phi}\right)=p_{1}\left(k_{\mu}+\frac{1}{2}, k_{\Phi}+\frac{1}{2}\right)$. One can observe that $p_{1}+p_{2}=1$. The function $g_{2}(x)$ is introduced in (B9).

Let us consider the limit of zero temperature, or $\beta \rightarrow \infty$. The expression (58) becomes exact in this limit. Since $b_{1}$ and $b_{2}$ are linear in time, we can perform easily all time integrations. Thus, the averaging over zero modes effectively results in replacing $\tilde{N} \rightarrow$ $2 f\left(k_{\mu}+\frac{1}{2} \delta_{\mu 1(2)}\right)$ and $\tilde{J} \rightarrow 2 f\left(k_{\Phi}+\frac{1}{2} \delta_{\Phi 1(2)}\right)$, where $\delta_{\mu 1}=\delta_{\Phi 1}=0$ and $\delta_{\mu 2}=\delta_{\Phi 2}=1$, which assumes further summation over the different topological realizations (1 and 2) of the ground state with the weight factors $p_{1}$ and $p_{2}$. For $\beta^{-1} \ll \omega_{0}$ the latter approximately equal

$$
\begin{aligned}
& p_{1}\left(k_{\mu}, k_{\Phi}\right)=\Theta\left(-\tilde{\nu} g_{2}\left(k_{\mu}\right)-\lambda g_{2}\left(k_{\Phi}\right)\right), \\
& p_{2}\left(k_{\mu}, k_{\Phi}\right)=\Theta\left(\tilde{\nu} g_{2}\left(k_{\mu}\right)+\lambda g_{2}\left(k_{\Phi}\right)\right),
\end{aligned}
$$

and play the role of projectors which divide the elementary cell $\left(k_{\Phi}, k_{\mu}\right) \in\left[-\frac{1}{2}, \frac{1}{2}\right] \times\left[-\frac{1}{2}, \frac{1}{2}\right]$ into two areas (topological sectors).

Let us analyze such a partition of the elementary cell and consider the (upper right) quadrant defined by $0<$ $k_{\mu}<\frac{1}{2}$ and $0<k_{\Phi}<\frac{1}{2}$. The function $g_{2}(x)=x-1 / 4$

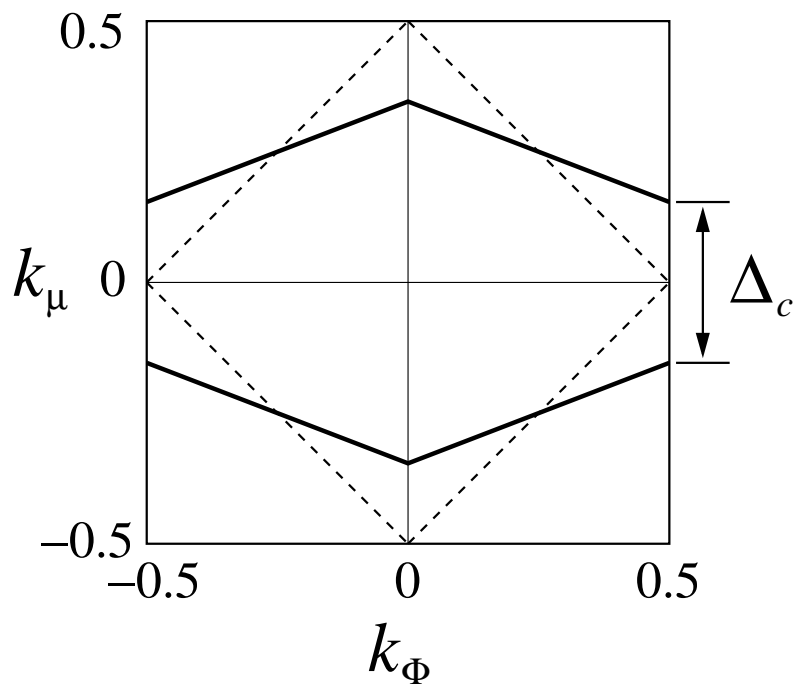

FIG. 4: Shift of the conductance peaks (solid lines) due to the charging energy. The dashed lines correspond to the noninteracting case $\left(\Delta_{c}=0\right)$.

for $0<x<1 / 2$, and therefore the border between the areas of $p_{1}$ and $p_{2}$ is given by the equation

$$
\tilde{\nu} k_{\mu}+\lambda k_{\Phi}=\frac{\tilde{\nu}+\lambda}{4} .
$$

For repulsive interactions and, moreover, in the presence of $E_{c} \neq 0$ the relation $\tilde{\nu} / \lambda>1$ is fulfilled.

We can establish the borders between the topological sectors $p_{1}$ and $p_{2}$ in the other quadrants by mirroring Eq. (63) with respect to the $k_{\mu}$ axis, $k_{\Phi}$ axis, or both. Thus, we obtain that the area of the projector $p_{1}$ is the inner part of the elementary cell bounded by the hexagon (see Fig. (4). Respectively, the outer part is the area of $p_{2}$.

Let us now analyze the conductance in the upper right quadrant. In the inner part $p_{1}$ only the zero harmonic $(p=0)$ of $A_{R}^{+}$becomes divergent near the border line (63). In the outer part $p_{2}$ the zero harmonic $(p=0)$ of $A_{R}^{-}$is divergent near the border line (63). From both sides of the latter the conductance behaves like

$$
G \propto \frac{1}{\left[\tilde{\nu} k_{\mu}+\lambda k_{\Phi}-\frac{\tilde{\nu}+\lambda}{4}\right]^{2}} .
$$

This is an expected result as well as the fact that the pole of the conductance (condition for the resonant tunneling of an electron) matches with a transition $p_{1} \rightarrow p_{2}$ from one topological sector to another.

In order to identify the positions of the conductance peaks, it appears sufficient to consider just the zero harmonics $p=0$ of the functions $A_{\eta}^{ \pm}$, because the higher ones $(p \geq 1)$ do not have any poles at all.

It is instructive to derive the conductance in the noninteracting limit $\tilde{\nu}=\lambda=1$. Using the identity

$$
\frac{\pi}{\sin \pi x}=\sum_{p=-\infty}^{\infty} \frac{(-1)^{p}}{p+x},
$$


one can show that

$$
G=\frac{e^{2}}{2 \pi} \frac{\Gamma_{l} \Gamma_{r}}{\omega_{0}^{2}}\left[\frac{\pi}{\sin \left(\frac{\Delta \mu}{\omega_{0}}+k_{\Phi}\right) \pi}+\frac{\pi}{\sin \left(\frac{\Delta \mu}{\omega_{0}}-k_{\Phi}\right) \pi}\right]^{2} .
$$

This result can be recovered in the scattering matrix approach, if the width of the Breit-Wigner resonance in (6) is neglected. The expression (66) suggests that the resonant condition can be satisfied at any $k_{\mu}$ by tuning the magnetic flux $k_{\Phi}$.

As was discussed in Ref. 16, the main qualitative feature imposed by an electron-electron interaction and/or charging energy is the opening of a window at certain values of $k_{\mu}$ inside which the resonant condition is never met. This situation is shown in Fig. [4 and the corresponding gap value equals $\Delta_{c}=\frac{1}{2}\left(1-\frac{\lambda}{\tilde{\nu}}\right)$.

\section{B. Spinful case}

In order to implement an averaging similar to (53) in the spinful cases, it is necessary to calculate first the partition function $\operatorname{Tr}\left(e^{-\beta H_{0}}\right)$. The trace operation is now understood as a summation over all integer values of $N_{R+}$, $N_{L+}, N_{R-}$, and $N_{L-}$. However, in this basis the Hamiltonian (48) is not diagonal, and we have to use the basis (49), (50) instead. The summation rules for the latter have been formulated, for instance, in Refs. 252829 . Applying them, one can find that the partition function is proportional to

$$
\begin{aligned}
& \sum_{i=1}^{16}\left[\theta_{3}\left(\pi k_{\mu i}, e^{-\frac{\pi^{2}}{2 \beta \omega_{0} \tilde{\nu}_{c}}}\right) \theta_{3}\left(\pi k_{\Phi i}, e^{-\frac{\pi^{2}}{2 \beta \omega_{0} \lambda_{c}}}\right)\right. \\
& \left.\times \theta_{3}\left(\pi k_{B i}, e^{-\frac{\pi^{2}}{2 \beta \omega_{0} \nu_{s}}}\right) \theta_{3}\left(\pi k_{R i}, e^{-\frac{\pi^{2}}{2 \beta \omega_{0} \lambda_{s}}}\right)\right],
\end{aligned}
$$

where $k_{X i}=k_{X}+\frac{1}{4} \delta_{X i}(X=\mu, \Phi, B, R)$ and the summation is performed over 16 topological sectors. The latter are specified by $\delta_{X i}$ given in the table

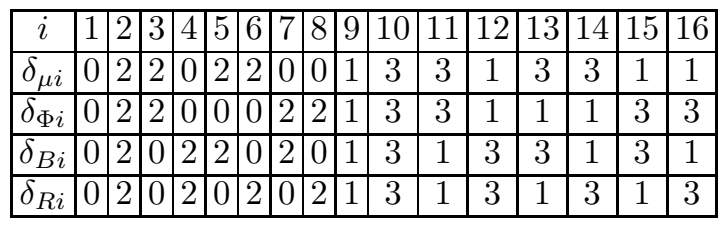

One can define 16 functions $(i=1, \ldots, 16)$

$$
p_{i}\left(k_{X}\right)=p_{1}\left(k_{X i}\right),
$$

where $p_{1}\left(k_{X}\right)$ equals to

$$
\begin{aligned}
& \frac{1}{1+\sum_{j=2}^{16} \frac{\theta_{3}\left(\pi k_{\mu j}\right)}{\theta_{3}\left(\pi k_{\mu}\right)} \frac{\theta_{3}\left(\pi k_{\Phi j}\right)}{\theta_{3}\left(\pi k_{\Phi}\right)} \frac{\theta_{3}\left(\pi k_{B j}\right)}{\theta_{3}\left(\pi k_{B}\right)} \frac{\theta_{3}\left(\pi k_{R j}\right)}{\theta_{3}\left(\pi k_{R}\right)}} \\
& \approx \frac{1}{1+\sum_{j=2}^{16} e^{2 \beta \omega_{0} B_{j}\left(k_{X}\right)}},
\end{aligned}
$$

and the functions $B_{j}\left(k_{X}\right)$ are introduced in Appendix $\mathrm{B}$
The functions (68) satisfy the identity

$$
\sum_{i=1}^{16} p_{i}\left(k_{X}\right)=1
$$

At low temperatures $\beta^{-1} \ll \omega_{0}$ we have an approximate relation

$$
p_{1}\left(k_{X}\right)=\prod_{j=2}^{16} \Theta\left(-B_{j}\left(k_{X}\right)\right)
$$

and the functions $p_{i}\left(k_{X}\right)$ become the projectors which divide the elementary cell $k_{X} \in\left[-\frac{1}{2}, \frac{1}{2}\right] \times \cdots \times\left[-\frac{1}{2}, \frac{1}{2}\right]$ in the four-dimensional parameter space into 16 topological sectors.

We can now formulate the rule which prescribes how to evaluate the average over zero modes in (40): it is necessary to replace

$$
\begin{aligned}
& \tilde{N}_{c} \rightarrow 4 f\left(k_{\mu i}\right), \quad \tilde{J}_{c} \rightarrow 4 f\left(k_{\Phi i}\right), \\
& \tilde{N}_{s} \rightarrow 4 f\left(k_{B i}\right), \quad \tilde{J}_{s} \rightarrow 4 f\left(k_{R i}\right),
\end{aligned}
$$

and to sum over $i=1, \ldots, 16$ topological realizations of the ground state with the weight functions $p_{i}\left(k_{X}\right)$.

Once this procedure is implemented, it becomes sufficient to consider just the zero harmonics $\left(p_{c}=p_{s}=0\right)$ of the functions $A_{\eta \sigma}^{ \pm}$, Eq. (46), for establishing the positions of the conductance maxima. In this respect there exists a full analogy with the spinless case, and we refer to the corresponding discussion in the previous subsection.

In the framework of the developed formalism it is possible to study the influence of the TLL interaction on the distribution of the conductance peaks in the presence of magnetic flux and SO coupling. The charging effects are also naturally incorporated, and the charging energy $E_{c}$ plays a role similar to that of the TLL parameter $\nu_{c}$. They are both combined into $\tilde{\nu}_{c}$ [see Eq. [45)], and therefore the effects produced by each of them are analogous. Let us then fix $\nu_{c}=1$ and vary $E_{c}$. In Fig. 5 we show the elementary cells of the conductance contour plot in the $\left(k_{\Phi}, k_{\mu}\right)$ plane for $k_{R}=0.1$ and different values of the charging energy. The TLL parameters are $\nu_{c, s}=\lambda_{c, s}=1$. One can see how the separate effects of SO coupling and charging energy (shown in Figs. 3 and 4. respectively) merge together.

In experiments the usual tuning parameters are $\Phi$ and $V_{g}$. The parameter $V_{g}$ appears in the theoretical model through both $k_{R}$ and $k_{\mu}$. The Rashba coupling constant depends on an applied gate voltage $e^{\underline{7}}$, which is modeled by $\alpha_{R}=\alpha_{R}^{0}-\frac{\pi}{m^{*} L} \kappa v_{g}$, where $v_{g}=\frac{e V_{g}}{\omega_{0}}$ and $\kappa>0$ is a dimensionless coefficient. The degeneracy point in gate voltage at which the Rashba coupling $\alpha_{R}$ vanishes is defined by $v_{g}^{0} \equiv v_{g}\left(\alpha_{R}=0\right)=\frac{m^{*} L \alpha_{R}^{0}}{\kappa \pi}$. Introducing the departure from the degeneracy point $\Delta v_{g}=v_{g}-v_{g}^{0}$, we then express

$$
k_{R}\left(\Delta v_{g}\right)=\frac{1}{2}\left[\sqrt{1+\kappa^{2}\left(\Delta v_{g}\right)^{2}}-1\right]
$$




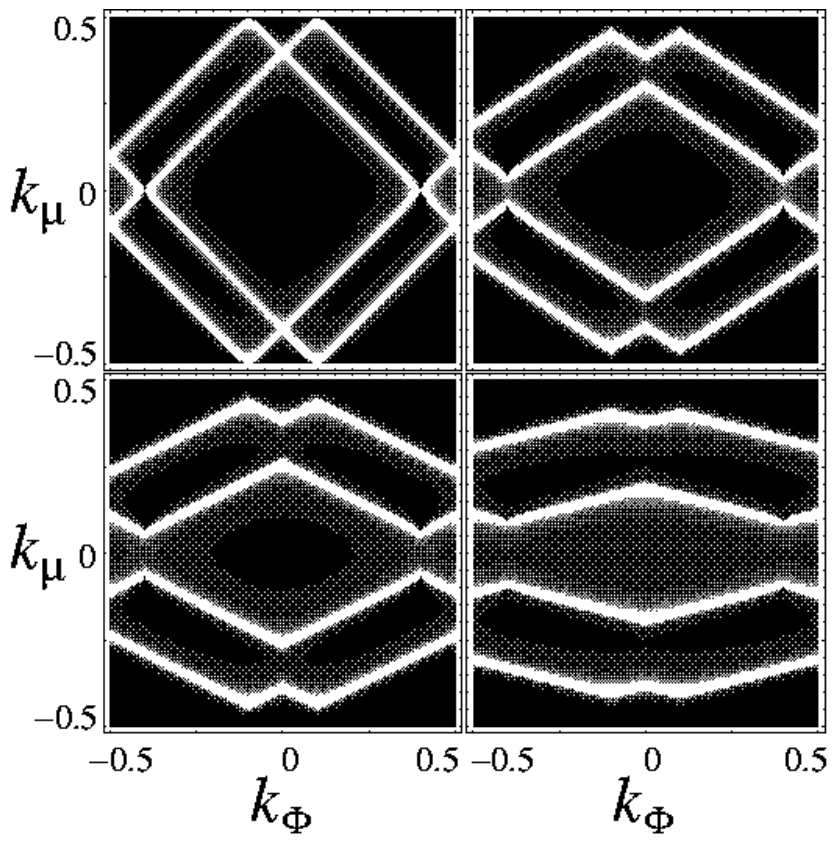

FIG. 5: Conductance peaks for $k_{R}=0.1$ and different values of the charging energy $8 E_{c} / \omega_{0}=0.0$ (upper left), 0.5 (upper right), 1.0 (bottom left), 3.0 (bottom right). The TLL parameters are $\nu_{c, s}=\lambda_{c, s}=1$.

and

$$
k_{\mu}\left(\Delta v_{g}\right)=\frac{\Delta v_{g}+v^{\prime}}{1+8 E_{c} / \omega_{0}} .
$$

Here $v^{\prime}=v_{g}^{0}+\frac{\Delta \mu}{\omega_{0}}+N_{0}-\frac{1}{2}$ determines the shift of the whole pattern; we may put at will $v^{\prime}=0$.

In Fig. 6] we demonstrate the influence of the charging effects in $\left(k_{\Phi}, \Delta v_{g}\right)$ plane. The values of $E_{c}$ and $\nu_{c, s}$, $\lambda_{c, s}$ are the same as in Fig. [5] We observe that upon enhancement of the charging energy the gap opens near $\Delta v_{g}=0$. Due to the presence of the gate-dependent SO coupling, the pattern of conductance maxima is more complicated than that discussed in Ref. 16.

\section{DISCUSSION AND CONCLUSION}

In this work we have studied the tunneling conductance of a mesoscopic one-dimensional ring attached to two Fermi reservoirs. The interaction inside the ring is described by the Tomonaga-Lutinger liquid. The bosonization approach which is usually adopted for the study of such model allows us to include the flux and gate-voltage dependence as well as the influence of SO coupling. It is remarkable that all these externally tuned parameters appear in the topological sector of the bosonized theory. Therefore, the accurate treatment of zero modes is required in order to describe the mesoscopic phenomena at low temperatures $\beta^{-1} \ll \omega_{0}$.

Using the Keldysh formalism we have performed the calculation of a linear conductance in the limit of weak

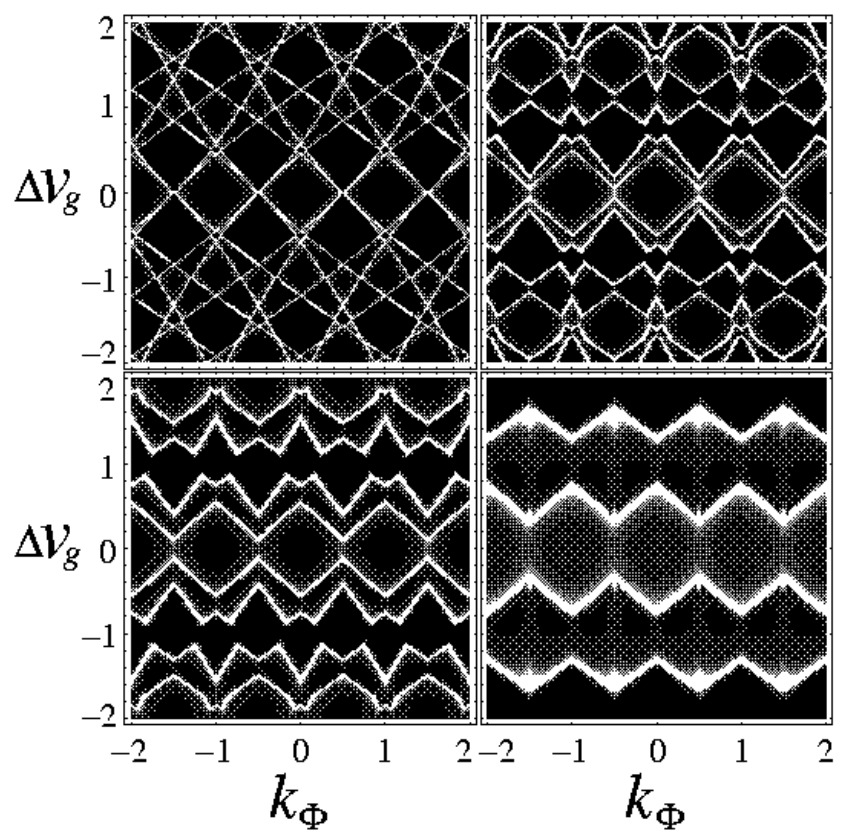

FIG. 6: Conductance peaks for $\kappa=1$ and different values of the charging energy $8 E_{c} / \omega_{0}=0.0$ (upper left), 0.5 (upper right), 1.0 (bottom left), 3.0 (bottom right).

tunneling between the leads and the ring. The real-time approach allows one to obtain asymptotic results for the distribution of the conductance peaks in the low temperature limit. Although the perturbative expansion of the conductance in the tunneling strength is not very well suited for a description of a shape of conductance peaks, it is nevertheless quite efficient for establishing their positions. The topological origin of the peaks' distribution alludes to its robustness upon small modifications of the model.

We have studied the patterns of the conductance maxima at nonzero spin-orbit coupling as a function of magnetic flux and gate voltage. The Tomonaga-Luttinger interaction and the charging energy have been seen to contribute in analogous way. In rings of reduced size the account of charging effects might appear more experimentally motivated, and therefore we concentrated on their study. In particular, we have made a theoretical prediction for the distribution of the conductance peaks in the presence of both the charging energy and the spinorbit coupling. We observed an interesting interplay between both effects. The SO coupling lifts the degeneracy of the conduction peaks, and the charging energy opens a gap centered at the remaining points of the degeneracy in question. The value of this gap is proportional to the charging energy. When the latter becomes very large, the Rashba effect is less pronounced. The pattern of the conductance peaks then approaches the form of hexagonal honeycombs which is typical to spinless fermions.

In conclusion, we have described the interplay between Coulomb blockade and Aharonov-Bohm and AharonovCasher effects for the different values of the charging en- 
ergy, magnetic flux, and spin-orbit coupling, as is manifested in the contour plots of the tunneling conductance.

\section{Acknowledgments}

We would like to thank Dmitry Bagrets, Thierry Champel, Ilya Krive, and Gerd Schön for fruitful discussions. M.P. was supported by the DFG Center for Functional Nanostructures at the University of Karlsruhe. V.G. was supported by the Swiss National Science Foundation.

\section{APPENDIX A: CORRELATION FUNCTIONS OF THE TOMONAGA-LUTTINGER MODEL}

Let us consider for simplicity the spinless case. The canonical transformation which solves the two-channel TLL model is

$$
\left(\begin{array}{l}
d_{1 k} \\
d_{2 k}^{\dagger}
\end{array}\right)=\left(\begin{array}{ll}
u_{+} & u_{-} \\
u_{-} & u_{+}
\end{array}\right)\left(\begin{array}{c}
b_{R k} \\
b_{L k}^{\dagger}
\end{array}\right)
$$

where $u_{ \pm}=\sqrt{(\gamma \pm 1) / 2}$

The explicit form of the time evolution of the bosonized fields reads

$$
\begin{aligned}
& \psi_{\eta}^{b}(x, t)=\frac{1}{\sqrt{L \tilde{\alpha}}} e^{-i \sqrt{2 \pi} \phi_{\eta}(x, t)}, \\
& \phi_{\eta}(x, t)=i \sum_{k=1}^{\infty} \frac{e^{-\frac{1}{2} \tilde{\alpha} k}}{\sqrt{2 \pi k}} \\
& \times\left[u_{\eta} D_{1 k}(x-\omega t)-u_{-\eta} D_{2 k}(x+\omega t)\right],
\end{aligned}
$$

where

$$
\begin{aligned}
& D_{1 k}=e^{i k x} d_{1 k}-e^{-i k x} d_{1 k}^{\dagger} \\
& D_{2 k}=e^{i k x} d_{2 k}^{\dagger}-e^{-i k x} d_{2 k}
\end{aligned}
$$

Let us consider the correlation function

$$
\begin{aligned}
\left\langle\psi_{\eta}^{b}(x, t) \psi_{\eta}^{b \dagger}\right\rangle & =e^{-\pi\left\langle\left(\phi_{\eta}(x, t)-\phi_{\eta}\right)^{2}\right\rangle+\pi\left[\phi_{\eta}(x, t), \phi_{\eta}\right]} \\
& =e^{-u_{+}^{2}(\pi \mathcal{D}(\eta x-\omega t)-i \chi(\eta x-\omega t))} \\
& \times e^{-u_{-}^{2}(\pi \mathcal{D}(\eta x+\omega t)+i \chi(\eta x+\omega t))}
\end{aligned}
$$

where

$$
\begin{aligned}
& \mathcal{D}(x)=\frac{1}{\pi} \sum_{k=1}^{\infty} \frac{1-\cos k x}{k}\left(\frac{2}{e^{\beta \omega k}-1}+e^{-\tilde{\alpha} k}\right) \\
& \chi(x)=\sum_{k=1}^{\infty} \frac{\sin k x}{k} e^{-\tilde{\alpha} k}=\frac{1}{2 i} \ln \frac{1-e^{-i x-\tilde{\alpha}}}{1-e^{i x-\tilde{\alpha}}}
\end{aligned}
$$

are the periodic functions of $x$. Obviously, the function (A6) is also periodic in real time, and therefore it can be expanded in a Fourier series with the frequency $\omega$.
In the zero-temperature limit $\beta \rightarrow \infty$ the temperaturedependent part of $\mathcal{D}(x)$ can be discarded, and we obtain

$$
\mathcal{D}(x)=-\frac{1}{2 \pi} \ln \frac{\left(1-e^{-\tilde{\alpha}}\right)^{2}}{\left(1-e^{-i x-\tilde{\alpha}}\right)\left(1-e^{i x-\tilde{\alpha}}\right)} .
$$

Hence, the correlation function A6 is equal to

$$
\left(\frac{1-e^{-\tilde{\alpha}}}{1-e^{i(\eta x-\omega t+i \tilde{\alpha})}}\right)^{u_{+}^{2}}\left(\frac{1-e^{-\tilde{\alpha}}}{1-e^{-i(\eta x+\omega t-i \tilde{\alpha})}}\right)^{u_{-}^{2}} .
$$

For $x=\pi$ we have

$$
\left\langle\psi_{\eta}^{b}(\pi, t) \psi_{\eta}^{b \dagger}\right\rangle=\frac{1}{L \tilde{\alpha}}\left(\frac{1-e^{-\tilde{\alpha}}}{1+e^{-i \omega t-\tilde{\alpha}}}\right)^{\gamma} .
$$

Its Fourier expansion (35) is equivalent to the Taylor expansion of the analytic function $(1+z)^{-\gamma}$ for $|z|<1$. Therefore the expansion (35) contains only non-negative Fourier harmonics $(p \geq 0)$ with the coefficients

$$
\begin{aligned}
g_{p}(\gamma) & =\frac{(-1)^{p} \Gamma(p+\gamma)}{\Gamma(\gamma) \Gamma(p+1)} \frac{\left(1-e^{-\tilde{\alpha}}\right)^{\gamma}}{L \tilde{\alpha}} e^{-p \tilde{\alpha}} \\
& \approx \frac{(-1)^{p} \Gamma(p+\gamma)}{\Gamma(\gamma) \Gamma(p+1)} \frac{\tilde{\alpha}^{\gamma-1}}{L} .
\end{aligned}
$$

Computation of a correlation function in the spinful case is analogous. A new feature arising in this case is the double time periodicity of correlation functions with frequencies $\omega_{c}$ and $\omega_{s}$, which are in general incommensurate.

\section{APPENDIX B: JACOBIAN $\Theta$ FUNCTIONS}

The Jacobian function $\theta_{3}$ is defined by

$$
\theta_{3}(z, q)=1+2 \sum_{n=1}^{\infty} q^{n^{2}} \cos 2 n z
$$

and the Jacobian function $\theta_{4}$ can be expressed as

$$
\theta_{4}(z, q)=\theta_{3}\left(z+\frac{\pi}{2}, q\right)
$$

Both functions are periodic under the shift $z \rightarrow z+\pi$.

Making a Poisson summation, one can prove that

$$
\sum_{k=-\infty}^{\infty} e^{-a(k+z)^{2}}=\sqrt{\frac{\pi}{a}} \theta_{3}\left(\pi z, e^{-\pi^{2} / a}\right)
$$

Using the expression for the ratio of two $\theta_{3}$ functions,

$\ln \frac{\theta_{3}\left(z_{1}+z_{2}, q\right)}{\theta_{3}\left(z_{1}-z_{2}, q\right)}=4 \sum_{n=1}^{\infty} \frac{(-1)^{n}}{n} \frac{q^{n}}{1-q^{2 n}} \sin 2 n z_{1} \sin 2 n z_{2}$, 
one can establish that

$$
\lim _{\beta \rightarrow \infty} \frac{\theta_{3}\left(\pi\left(x+\frac{i b}{\beta \omega_{0} \lambda}\right), e^{-\frac{\pi^{2}}{2 \beta \omega_{0} \lambda}}\right)}{\theta_{3}\left(\pi x, e^{-\frac{\pi^{2}}{2 \beta \omega_{0} \lambda}}\right)}=e^{4 i b f(x)},
$$

where $f(x)$ is a sawtooth function introduced in (59), as well as that

$$
\lim _{\beta \rightarrow \infty} \frac{\theta_{3}\left(\pi\left(x+\frac{m_{1}}{4}\right), e^{-\frac{\pi^{2}}{2 \beta \omega_{0} \lambda}}\right)}{\theta_{3}\left(\pi\left(x+\frac{m_{2}}{4}\right), e^{-\frac{\pi^{2}}{2 \beta \omega_{0} \lambda}}\right)} \approx e^{2 \beta \omega_{0} \lambda g_{m_{1} m_{2}}(x)}
$$

where $m_{1}, m_{2}=0,1,2,3$ and

$$
\begin{aligned}
& g_{m_{1} m_{2}}(x)=\sum_{n=1}^{\infty} \frac{(-1)^{n}}{\pi^{2} n^{2}} \\
& \times\left[\cos \frac{\pi n}{2}\left(4 x+m_{2}\right)-\cos \frac{\pi n}{2}\left(4 x+m_{1}\right)\right] .
\end{aligned}
$$

In the Fourier series (B7) one can recognize the functions

$$
\begin{aligned}
g_{1}(x) & \equiv g_{10}(x)=-g_{01}(x) \\
& =\left|\left\{\frac{1}{2}+x\right\}-\frac{3}{4}\right|+\frac{1}{2}\left\{\frac{1}{2}+x\right\}-\frac{9}{16}, \\
g_{2}(x) & \equiv g_{20}(x)=-g_{02}(x) \\
& =-\left|\{x\}-\frac{1}{2}\right|+\frac{1}{4}, \\
g_{3}(x) & \equiv g_{30}(x)=-g_{03}(x) \\
& =\left|\left\{\frac{1}{2}-x\right\}-\frac{3}{4}\right|+\frac{1}{2}\left\{\frac{1}{2}-x\right\}-\frac{9}{16},
\end{aligned}
$$

1 Y. Aharonov and D. Bohm, Phys. Rev. 115, 485 (1959).

${ }^{2}$ Y. Aharonov and A. Casher, Phys. Rev. Lett. 53, 319 (1984).

3 M. König et al., Phys. Rev. Lett. 96, 076804 (2006).

4 A. F. Morpurgo, J. P. Heida, T. M. Klapwijk, B. J. van Wees, and G. Borghs, Phys. Rev. Lett. 80, 1050 (1998). See also H. De Raedt, Phys. Rev. Lett. 83, 1700 (1999); A. F. Morpurgo, J. P. Heida, T. M. Klapwijk, B. J. van Wees, and G. Borghs, Phys. Rev. Lett. 83, 1701 (1999).

5 T. Bergsten, T. Kobayashi, Y. Sekine, and J. Nitta, cond-mat/0512264

6 T. Koga, Y. Sekine, and J. Nitta, cond-mat/0504743

7 J. Nitta, T. Akazaki, H. Takayanagi, and T. Enoki, Phys. Rev. Lett. 78, 1335 (1997).

8 Yu. A. Bychkov and E. I. Rashba, J. Phys. C 17, 6039 (1984).

9 S. Datta and B. Das, Appl. Phys. Lett. 56, 665 (1990).

10 M. Büttiker, Y. Imry, and M. Ya. Azbel, Phys. Rev. A 30 1982 (1984); M. Büttiker, Y. Imry, R. Landauer, and S. Pinhas, Phys. Rev. B 31, 6207 (1985).

11 A. Stern, Phys. Rev. Lett. 68, 1022 (1992).

12 A. G. Aronov and Y. B. Lyanda-Geller, Phys. Rev. Lett. where $\{x\} \equiv(x \bmod 1)$ is a fractional part of $x$. The function $\{x\}$ has a period 1 and possesses a property $\{-x\}=1-\{x\}$. One can notice that $g_{3}(x)=g_{1}(-x)$.

The other functions $g_{m_{1} m_{2}}$ are also expressed in terms of $g_{1}, g_{2}, g_{3}$ :

$$
\begin{aligned}
& g_{12}(x)=-g_{21}(x)=g_{3}(x-1 / 2), \\
& g_{32}(x)=-g_{23}(x)=g_{1}(x+1 / 2), \\
& g_{31}(x)=-g_{13}(x)=g_{2}(x+1 / 4) .
\end{aligned}
$$

We also define the following functions:

$$
\begin{aligned}
B_{2} & =\tilde{\nu}_{c} g_{2}\left(k_{\mu}\right)+\lambda_{c} g_{2}\left(k_{\Phi}\right)+\nu_{s} g_{2}\left(k_{B}\right)+\lambda_{s} g_{2}\left(k_{R}\right), \\
B_{3} & =\tilde{\nu}_{c} g_{2}\left(k_{\mu}\right)+\lambda_{c} g_{2}\left(k_{\Phi}\right), \\
B_{4} & =\nu_{s} g_{2}\left(k_{B}\right)+\lambda_{s} g_{2}\left(k_{R}\right) \\
B_{5} & =\tilde{\nu}_{c} g_{2}\left(k_{\mu}\right)+\nu_{s} g_{2}\left(k_{B}\right), \\
B_{6} & =\tilde{\nu}_{c} g_{2}\left(k_{\mu}\right)+\lambda_{s} g_{2}\left(k_{R}\right), \\
B_{7} & =\lambda_{c} g_{2}\left(k_{\Phi}\right)+\nu_{s} g_{2}\left(k_{B}\right) \\
B_{8} & =\lambda_{c} g_{2}\left(k_{\Phi}\right)+\lambda_{s} g_{2}\left(k_{R}\right) \\
B_{9} & =\tilde{\nu}_{c} g_{1}\left(k_{\mu}\right)+\lambda_{c} g_{1}\left(k_{\Phi}\right)+\nu_{s} g_{1}\left(k_{B}\right)+\lambda_{s} g_{1}\left(k_{R}\right), \\
B_{10} & =\tilde{\nu}_{c} g_{3}\left(k_{\mu}\right)+\lambda_{c} g_{3}\left(k_{\Phi}\right)+\nu_{s} g_{3}\left(k_{B}\right)+\lambda_{s} g_{3}\left(k_{R}\right), \\
B_{11} & =\tilde{\nu}_{c} g_{3}\left(k_{\mu}\right)+\lambda_{c} g_{3}\left(k_{\Phi}\right)+\nu_{s} g_{1}\left(k_{B}\right)+\lambda_{s} g_{1}\left(k_{R}\right), \\
B_{12} & =\tilde{\nu}_{c} g_{1}\left(k_{\mu}\right)+\lambda_{c} g_{1}\left(k_{\Phi}\right)+\nu_{s} g_{3}\left(k_{B}\right)+\lambda_{s} g_{3}\left(k_{R}\right), \\
B_{13} & =\tilde{\nu}_{c} g_{3}\left(k_{\mu}\right)+\lambda_{c} g_{1}\left(k_{\Phi}\right)+\nu_{s} g_{3}\left(k_{B}\right)+\lambda_{s} g_{1}\left(k_{R}\right), \\
B_{14} & =\tilde{\nu}_{c} g_{3}\left(k_{\mu}\right)+\lambda_{c} g_{1}\left(k_{\Phi}\right)+\nu_{s} g_{1}\left(k_{B}\right)+\lambda_{s} g_{3}\left(k_{R}\right), \\
B_{15} & =\tilde{\nu}_{c} g_{1}\left(k_{\mu}\right)+\lambda_{c} g_{3}\left(k_{\Phi}\right)+\nu_{s} g_{3}\left(k_{B}\right)+\lambda_{s} g_{1}\left(k_{R}\right), \\
B_{16} & =\tilde{\nu}_{c} g_{1}\left(k_{\mu}\right)+\lambda_{c} g_{3}\left(k_{\Phi}\right)+\nu_{s} g_{1}\left(k_{B}\right)+\lambda_{s} g_{3}\left(k_{R}\right) .
\end{aligned}
$$

70, 343 (1993).

13 J. Nitta, F.E. Meijer, and H. Takayanagi, Appl. Phys. Lett. 75, 695 (1999).

14 D. Frustaglia and K. Richter, Phys. Rev. B 69, 235310 (2004).

15 B. Molnár, F. M. Peeters, and P. Vasilopoulos, Phys. Rev. B 69, 155335 (2004).

16 J. M. Kinaret, M. Jonson, R. I. Shekhter, and S. Eggert, Phys. Rev. B 57, 3777 (1998).

17 Y. Meir and N. S. Wingreen, Phys. Rev. Lett. 68, 2512 (1992).

18 C. L. Kane and M. P. A. Fisher, Phys. Rev. Lett. 68, 1220 (1992).

19 D. Boese, M. Governale, A. Rosch, and U. Zülicke, Phys. Rev. B 64, 085315 (2001).

${ }^{20}$ O. M. Auslaender, A. Yacobi, R. de Picciotto, K. W. Baldwin, L. N. Pfeiffer, and K. W. West, Science 295, 825 (2002).

21 A. Furusaki and N. Nagaosa, Phys. Rev. B 47, 3827 (1993).

22 A. Furusaki, Phys. Rev. B 57, 7141 (1998).

23 Yu. V. Nazarov and L.I. Glazman, Phys. Rev. Lett. 91, 126804 (2003). 
24 J. von Delft and H. Schoeller, Ann. Phys. (Leipzig) 7, 225 (1998).

25 R. Fazio, F. W. J. Hekking, and A. A. Odintsov, Phys. Rev. Lett. 74, 1843 (1995); Phys. Rev. B 53, 6653 (1996).

26 D. Loss, Phys. Rev. Lett. 69 , 343 (1992).

27 M. R. Geller and D. Loss, Phys. Rev. B 56, 9692 (1997).

28 M. V. Moskalets, Physica B 291, 350 (2000).
29 M. Pletyukhov and V. Gritsev, Phys. Rev. B 70, 165316 (2004).

30 I. V. Krive, A. M. Kadigrobov, R. I. Shekhter, and M. Jonson, Phys. Rev. B 71, 214516 (2005).

31 G. D. Mahan, Many-Particle Physics (Plenum Press, New York, 1990). 